\title{
Micafungin: a sulfated echinocandin
}

\author{
Seiji Hashimoto
}

Micafungin is the second approved antifungal agent in the echinocandin series and is now used worldwide in chemotherapy for life-threatening fungal infections. It is water-soluble and is semi-synthesized from the acylated cyclic hexapeptide FR901379, a natural product from the fungus Coleophoma empetri F-11899, through enzymatic deacylation of FR901379, followed by chemical reacylation with the optimized $\mathrm{N}$-acyl side chain. The water solubility of micafungin is ascribed to a sulfate moiety in the molecule. This feature differentiates micafungin from other echinocandin members. Micafungin is a potent inhibitor of

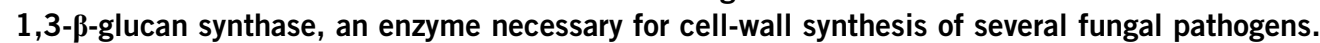
The Journal of Antibiotics (2009) 62, 27-35; doi:10.1038/ja.2008.3; published online 9 January 2009

Keywords: antifungal; echinocandin; FK463; 1,3- $\beta$-glucan synthase; micafungin

\section{INTRODUCTION}

Fungal infections cause not only superficial diseases such as athlete's foot and onychomycoses, but also life-threatening diseases. Serious deep-seated fungal infections caused by Candida spp., Aspergillus spp. and Cryptococcus neoformans are a threat to human health. Incidences of these systemic fungal infections have increased significantly over the past few years. The major reasons for this dramatic increase are the extensive use of broad-spectrum antibiotics and the growing number of immunocompromised patients with acquired immunodeficiency syndrome (AIDS), cancer and transplants. ${ }^{1,2}$

In the mid 1900s, few compounds, such as polyenes (for example, nystatin and amphotericin B) and flucytosine, were available for antifungal chemotherapy. Although the development of azole drugs started in the early 1970s, only a limited number of antifungal agents were available for treatment of life-threatening fungal infections. Moreover, the existing agents had disadvantages, such as the significant nephrotoxicity of amphotericin $\mathrm{B}^{3}$ and the emergence of resistance to the azoles. ${ }^{4}$ To overcome these defects, lipid formulations of polyenes were developed to reduce toxicity, and new triazoles (for example, voriconazole, ravuconazole and posaconazole) were developed to improve the antifungal spectra or susceptibility to azoleresistant isolates. ${ }^{5}$ Despite a number of therapeutic advancements, there was a need to develop a new class of antifungal agents with novel mechanisms of action.

The echinocandins were a new class of antifungal drugs developed for the first time since azoles. The first launched echinocandin was caspofungin acetate (Merck \& Co. Inc. (Merck), Readington, NJ, USA), followed by micafungin (Fujisawa Pharmaceutical Co., Ltd, now Astellas Pharma Co., Ltd, Fujisawa, Japan) and anidulafungin (Vicuron Pharmaceuticals Inc., now Pfizer Inc., New York, NY, USA), which was originally developed by Eli Lilly and Company (Indianapolis, IN, USA)
(Lilly) as LY 303366 and subsequently licensed to Vicuron (formerly Versicor) as VER-002. The approved echinocandins are synthetically modified lipopeptides that originate from natural compounds produced by filamentous fungi. The original anidulafungin, caspofungin and micafungin compounds were echinocandin B from Aspergillus nidulans var. echinulatus, ${ }^{6}$ pneumocandin B0 from Glarea lozoyensis ${ }^{7}$ and FR901379 from Coleophoma empetri, ${ }^{8}$ respectively.

Although natural echinocandins have potent antifungal activity in vitro, their structures were chemically altered to improve their absorption, distribution, metabolism and excretion characteristics. Such operations were initiated by Lilly on echinocandin B to yield cilofungin. ${ }^{9}$ This compound was subjected to Phase II clinical trials, but was abandoned due to toxicity. Further modification of the structure by converting the phenolic hydroxyl to a sodium phosphate ester produced the more soluble prodrug LY307853, which resulted in the active form, LY303366. ${ }^{10}$ Merck has produced MK-0991 using pneumocandin B0 as the starting material. ${ }^{11}$ MK-0991 likewise possesses increased water solubility. Other reviews on echinocandins or individual antifungal agents have reported their usefulness in clinical practice. ${ }^{12-16}$ This review describes the discovery and development of micafungin, focusing on the chemical diversity of echinocandins.

\section{DISCOVERY OF FR901379}

The seed compounds of micafungin, FR901379 and two related compounds (FR901381 and FR901382), were discovered at Fujisawa Pharmaceutical Co., Ltd in 1989 from the screening of approximately 6000 microbial broth samples (Figure 1). These new compounds were categorised as members of the echinocandin class of lipopeptides. Echinocandin B, pneumocandin B0 and other echinocandin lipopeptides are structurally characterized by a cyclic hexapeptide acylated with a long side chain, and have an excellent anti-Candida activity 


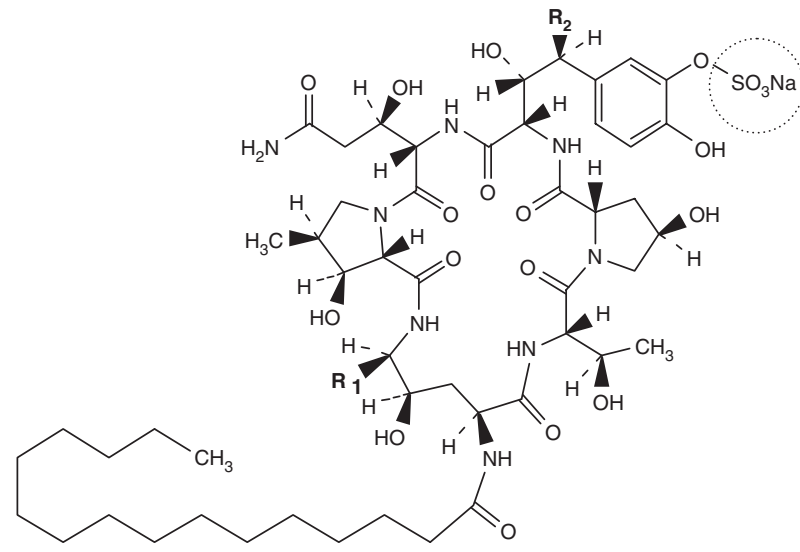

\begin{tabular}{crr} 
& R $_{1}$ & R $_{2}$ \\
\hline FR901379 & OH & OH \\
FR901381 & OH & OH \\
FR901382 & H & H \\
\hline
\end{tabular}

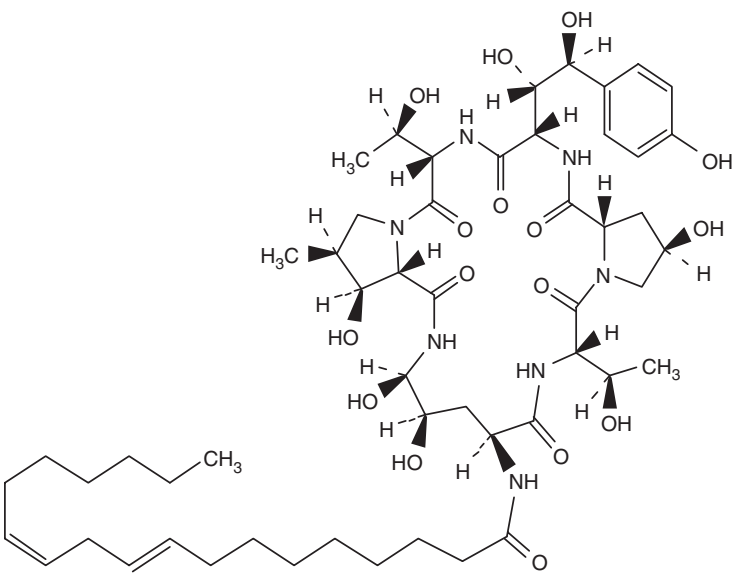

Echinocandin B

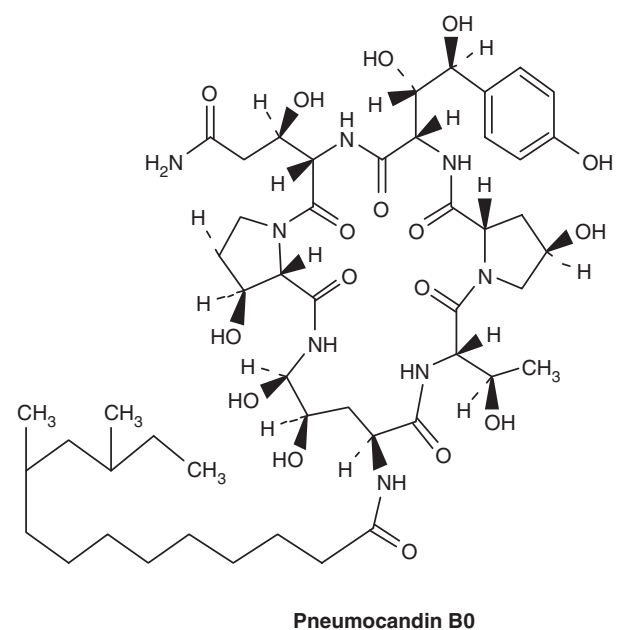

Figure 1 Structures of echinocandins.

attributed to selective inhibition of 1,3- $\beta$-glucan synthesis, although their intrinsic water insolubility is a major barrier for drug development. ${ }^{17-19}$ However, FR901379 and related compounds showed both high water solubility and a strong antifungal effect on Candida spp. ${ }^{20}$ The structural difference between FR901379 and the other echinocandins is that FR901379 has a sulfate moiety in its molecule (Figure 1, circled). This residue was speculated to be the basis for the high water solubility of FR901379 (soluble in water even at a concentration of $50 \mathrm{mg} \mathrm{ml}^{-1}$, a concentration at which other compounds have low solubility). To probe this hypothesis, FR901379 was digested with aryl sulfatase from Aerobacter aerogenes (Table 1), after which the water solubility of the desulfated molecule (FR133302) was decreased to $1 \mathrm{mg} \mathrm{ml}^{-1}$, even though the inhibitory activity on $1,3-\beta$-glucan synthase did not decrease markedly. ${ }^{21}$ This result indicated that the excellent water solubility of FR901379 was attributed to the sulfate moiety in its structure.

The $\mathrm{IC}_{50}$ value of FR901379 on 1,3- $\beta$-glucan synthase is $0.7 \mu \mathrm{g} \mathrm{ml}^{-1}$, which is superior to that of echinocandin B (Table 1). The in vitro antifungal activity of FR901379 and related compounds against both Candida albicans and A. fumigatus indicates a higher potency than that of aculeacin A (Table 2); however, it is only weakly active against $A$. fumigatus. None of these compounds show antifungal activity against $C$. neoformans. Table 3 shows the therapeutic effect of
FR901379 in a murine C. albicans infection model in which drugs were administered s.c. for four consecutive days. FR901379 and related compounds significantly prolonged the survival of infected mice. FR901379 was the most potent compound, with an $\mathrm{ED}_{50}$ value of $2.7 \mathrm{mg} \mathrm{kg}^{-1} 14$ days after the infection. This value was almost comparable to that of fluconazole. In spite of its potent antifungal activity and its good water solubility, FR901379 could not be developed further because of class-specific reticulocyte lysis at low concentrations (Table 4), although the lytic activity of FR901379 was weaker than that of amphotericin B.

The producer strain of FR901379, identified as C. empetri F-11899, was originally isolated from a soil sample collected at Iwaki City, Fukushima Prefecture, Japan. Its morphological characteristics were determined on the basis of cultures on sterilized azalea leaf affixed to a Miura's LCA plate, because the strain produced conidial structures on the leaf segment alone.

\section{GENERATION OF THE LEAD COMPOUND, FR131535}

When FR901379 was discovered by Fujisawa, both Merck and Lilly had already investigated this area of echinocandins. However, FR901379 had two advantages over other analogs. One was its excellent intrinsic water solubility, and the other was that the hemolytic activity of echinocandins was reduced by substituting the acyl side chain. As 
Table 1 Water solubility and inhibitory effect of echinocandins on 1,3- $\beta$-glucan synthase

\begin{tabular}{|c|c|c|}
\hline Compound & $\begin{array}{l}\text { Solubility in water } \\
\qquad\left(m g \mathrm{ml}^{-1}\right)\end{array}$ & $\begin{array}{c}\text { Inhibition of 1,3- } \beta \text {-glucan } \\
\text { synthase } 1 C_{50}\left(\mu g I^{-1}\right)\end{array}$ \\
\hline FR901379 & $>50$ & 0.7 \\
\hline Echinocandin B & 0.008 & 2.6 \\
\hline Cilofungin & 0.1 & nt \\
\hline
\end{tabular}

Abbreviation: nt, not tested.

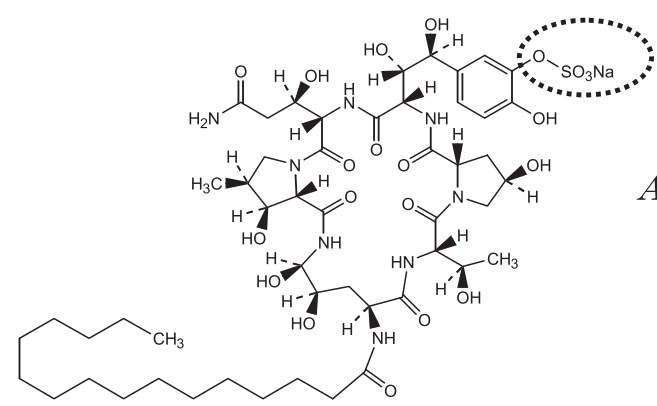

FR901379
Arylsulfatase from Aerobacter aerogenes

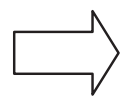

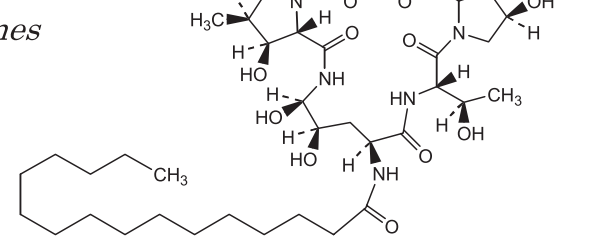

FR133302
Table 2 In vitro antifungal activity of FR901379 and related compounds

\begin{tabular}{lllll}
\hline \multirow{2}{*}{ Test organism } & \multicolumn{4}{c}{ IC $_{50}\left(\mu \mathrm{g} \mathrm{m}\right.$ I $\left.^{-1}\right)$} \\
\cline { 2 - 5 } & FR901379 & FR901381 & FR901382 & Aculeacin A \\
\hline Candida albicans FR578 & 0.008 & 0.008 & 0.008 & 0.008 \\
C. albicans FP582 & 0.025 & 0.015 & 0.03 & 0.06 \\
C. albicans FP629 & 0.008 & 0.004 & 0.008 & 0.015 \\
C. albicans FP633 & 0.025 & 0.025 & 0.03 & 0.06 \\
C. tropicalis YC118 & 0.025 & 0.05 & 0.015 & 0.31 \\
C. krusei YC109 & 0.16 & 0.16 & 0.16 & 0.62 \\
C. utilis YC123 & 0.03 & 0.003 & 0.003 & 0.06 \\
Aspergillus fumigatus FD050 & 1.9 & 1.6 & 0.62 & 2.5 \\
A. niger ATCC9642 & 0.03 & 0.03 & 0.03 & 2.5 \\
Cryptococcus neoformans & $>2.5$ & $>2.5$ & $>2.5$ & $>2.5$ \\
YC203 & & & & \\
\hline
\end{tabular}

Table 3 In vivo efficacy in a neutropenic mouse model of disseminated candidiasis

\begin{tabular}{lc}
\hline Compound & $E D_{50}\left(\mathrm{mg} \mathrm{kg}^{-1}\right)$ \\
\hline FR901379 & 2.7 \\
Aculeacin A & 6.4 \\
Fluconazole & 4.5 \\
\hline
\end{tabular}

Infection: Candida albicans FP633.

other compounds were not as soluble, Fujisawa's researchers focused on side-chain replacement, while keeping the sulfate group intact. Considering that the hemolytic activity may be related to the long alkyl side chain, they attempted to reduce the hemolytic activity of FR901379 by substituting the side chain, as Lilly's researchers had. ${ }^{22}$

FR901379 was treated with acylase from Actinoplanes utahensis to remove the palmitoyl group to yield FR179642, and a new acyl side
Table 4 Hemolytic activity of FR901379

\begin{tabular}{lc}
\hline Compound & $M L C^{\mathrm{a}}\left(\mu g \mathrm{I}^{-1}\right)$ \\
\hline FR901379 & 62 \\
Aculeacin A & 31 \\
Echinocandin B & 125 \\
Amphotericin B & 8 \\
\hline
\end{tabular}

aMinimum lytic concentration.

chain was prepared starting from 1-bromooctane and 4-hydroxybenzoic acid. Thus, 2,4,5-trichlorophenyl 4 -(n-octyloxy) benzoate was obtained from 4-(n-octyloxy) benzoic acid and 2,4,5-trichlorophenol using $N, N^{\prime}$-dicyclohexylcarbodiimide (DCC) in ether. The reacylation of FR179642 by the 2,4,5-trichlorophenyl active ester method yielded FR131535 (Figure 2). ${ }^{23}$ The water solubility of FR131535 was as high as that of FR901379, even after replacement of the acyl side chain. FR131535 inhibited 1,3- $\beta$-glucan synthase prepared from $C$. albicans 6406 with an $\mathrm{IC}_{50}$ value of $2.8 \mu \mathrm{g} \mathrm{ml}^{-1}$ in a non-competitive manner ( $\mathrm{Ki} 4.0 \mathrm{~mm}$ ) and exhibited potent activity against a variety of fungal species by the micro broth dilution method. The protective efficacy of FR131535 administered s.c. against a murine systemic infection with C. albicans was reported to be superior to those of echinocandin B and cilofungin, and almost as potent as fluconazole. Furthermore, FR131535 showed potent in vivo activity against A. fumigatus: the $\mathrm{ED}_{50}$ value of FR131535 was improved dramatically from 70 to $4.3 \mathrm{mg} \mathrm{kg}^{-1}$ (Table 5). Thus, FR131535 was the first echinocandin to show an effective anti-Aspergillus activity. The hemolytic activity of FR131535 had also been greatly reduced compared with that of FR901379 (Table 5). These findings led to extensive research on the optimization of the acyl side chain.

\section{LEAD COMPOUND OPTIMIZATION TO FK46324}

As the acyl side chain substitution led to the expansion of the antifungal spectrum to include Aspergillus spp., the relationship between the side-chain lipophilicity and antifungal activity was 


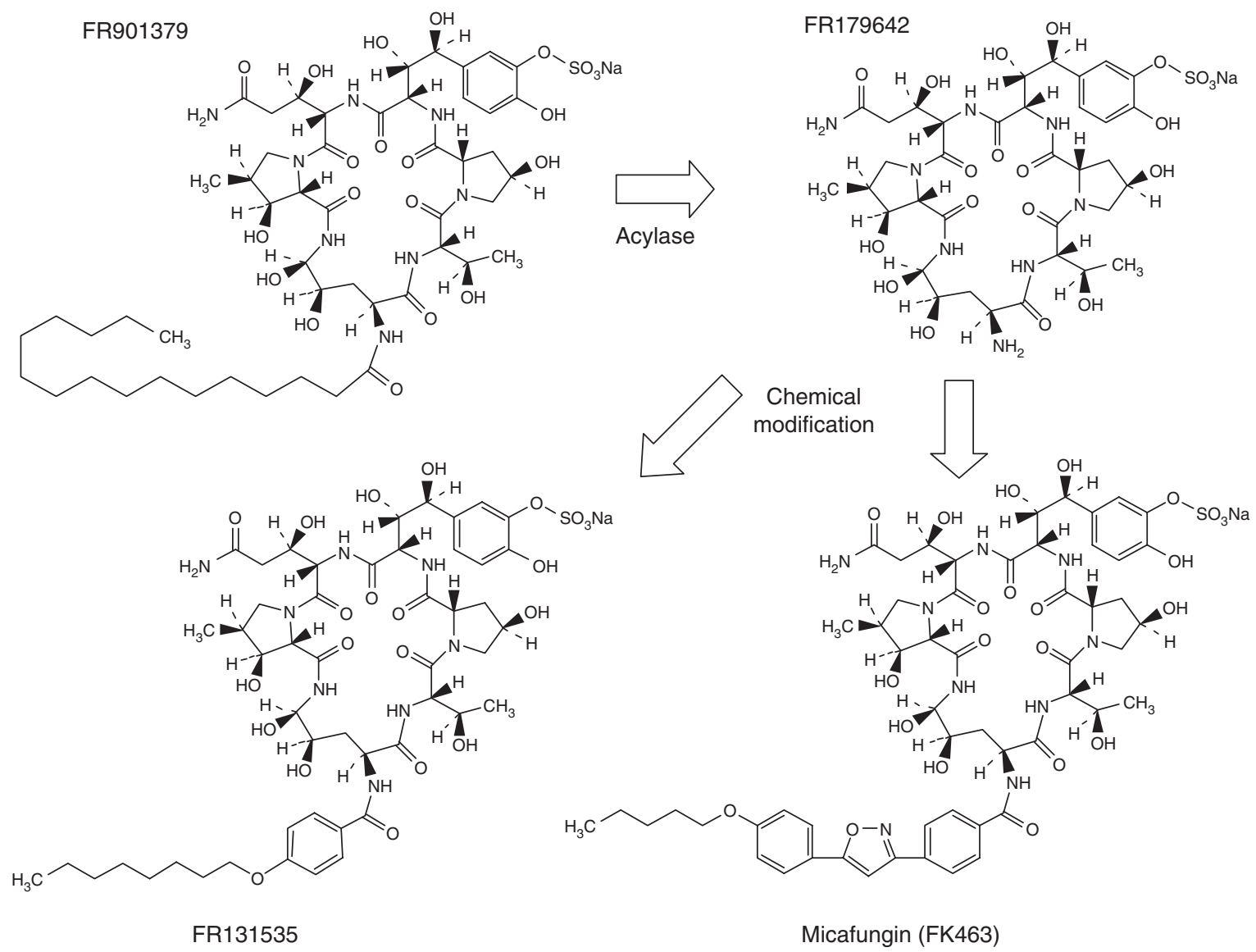

Figure 2 Semi-synthesis of FR131535 and micafungin (FK463).

Table 5 Influence of acyl side chain on MIC, ED $_{50}$ and hemolysis

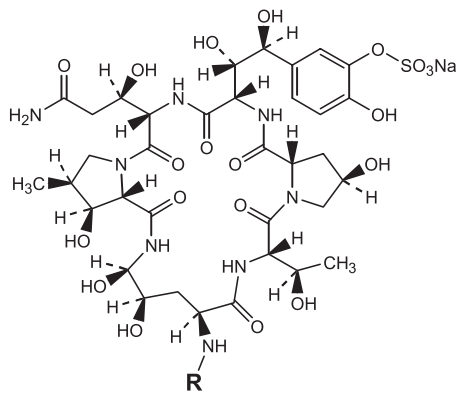

$\mathrm{R}=$

FR901379<smiles>CCCCCCCCCCCCCCCC(C)=O</smiles>

\section{FR131535}

$\mathrm{R}=$<smiles>CCCCCCCCOc1ccc(C(C)=O)cc1</smiles>

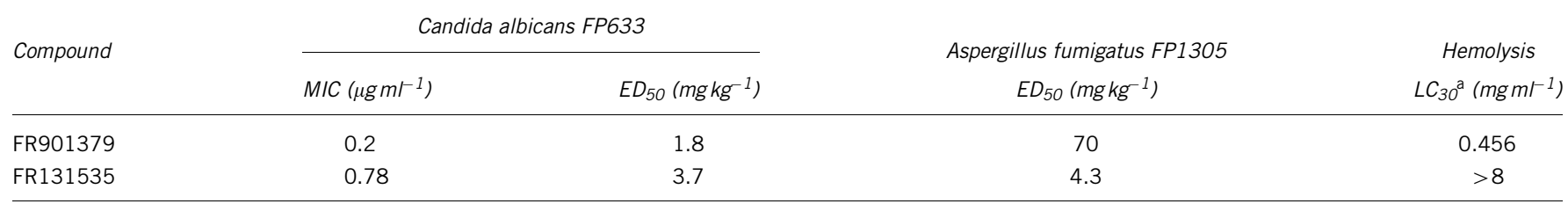

aLytic concentration $30 \%$.

examined. ${ }^{25,26}$ Naphthalene side chains, which are compact and amenable to modulation of lipophilicity, were chosen as the initial acyl side chains to be substituted. Meanwhile, the relationship between antifungal activities and hemolysis was examined by changing the lipophilicity by varying the length of the alkyl chains. The lipophilicity increase resulted in improved anti-Candida activity, which was most potent with an octyloxy group ( $n=7$; Figure $3 a)$. Hemolytic activity was enhanced in proportion to the length of the alkyl chains. To make the analog design more quantitative, the ClogP value [octanol-water partition coefficient (calculated value)] of the side chain was used to evaluate the correlation with anti-Candida activity. The strongest in vivo effect was observed in compounds with $\mathrm{C} \log \mathrm{P}$ values of 
a

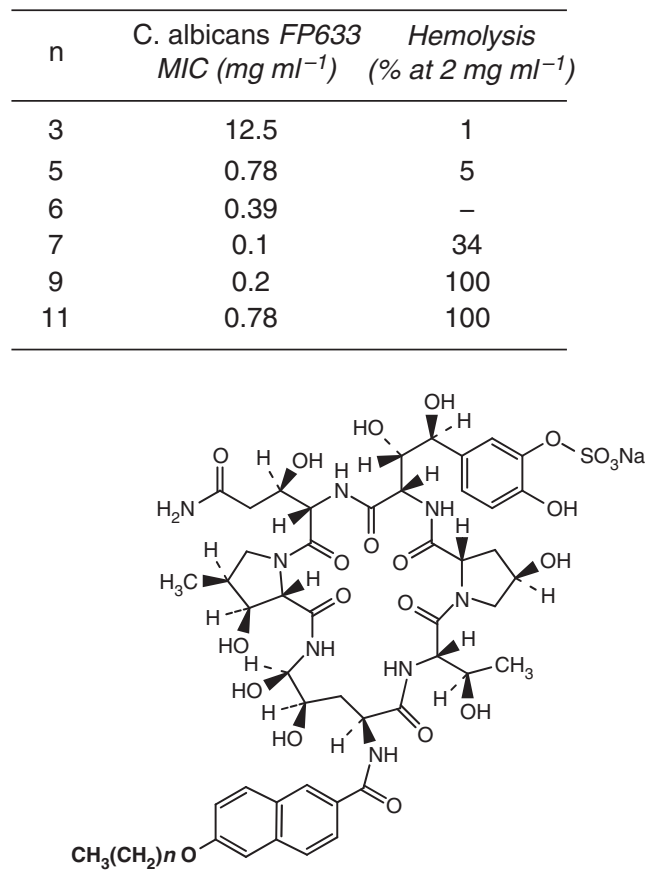

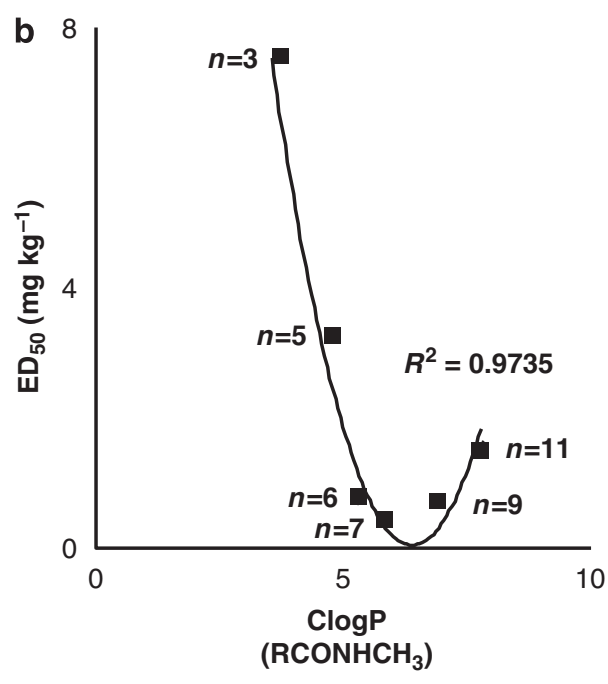

Figure 3 Effect of lipophilicity on MIC, hemolysis and $\mathrm{ED}_{50}$ : (a) length of alkyl chain; (b) $\mathrm{ClogP}$ of the side chain determined for $\mathrm{CH}_{3}\left(\mathrm{CH}_{2}\right)_{n} \mathrm{CONHCH}_{3}$.

Table 6 Key compounds in optimization for side chain

\begin{tabular}{|c|c|c|c|c|c|c|c|c|}
\hline \multirow{2}{*}{ Compound } & \multirow{2}{*}{ Side chain } & \multirow{2}{*}{$C \log P$} & \multicolumn{2}{|c|}{ Candida albicans FP633 } & \multicolumn{2}{|c|}{ Aspergillus fumigatus FP1305 } & \multicolumn{2}{|c|}{ Hemolysis } \\
\hline & & & $M I C\left(\mu g m I^{-1}\right)$ & Serum MIC $\left(\mu g \mathrm{~m}^{-1}\right)$ & $E D_{50^{\mathrm{a}}}\left(\mathrm{mg} \mathrm{kg}^{-1}\right)$ & $E D_{50}\left(m g \mathrm{~kg}^{-1}\right)$ & $L C_{30}{ }^{\mathrm{a}}\left(\mathrm{mg} \mathrm{m}^{-1}\right)$ & At $1 \mathrm{mg} \mathrm{m}^{-1}(\%)$ \\
\hline 1 & & 5.80 & 0.1 & 6.25 & 0.742 & 0.788 & 10 & \\
\hline 2 & & 5.37 & 0.2 & 6.25 & 0.658 & & 1.74 & \\
\hline 3 & & 6.14 & 0.025 & 1.56 & 0.447 & & 0.37 & 79 \\
\hline
\end{tabular}

a Lytic concentration $30 \%$.

approximately 6; therefore, the side chains were thereafter prepared by adjusting the ClogP value to approximately 6 (Figure $3 \mathrm{~b}$ ).

Conversion of a single benzene ring of FR131535 into a naphthalene ring improved anti-Candida activity, and further introduction of aromatic rings into the side chain was also effective (Table 6). Anti-Candida activity was improved as the number of benzene rings increased, and compound 3 showed the strongest MIC. Furthermore, compound 1, which had a naphthalene ring, showed a large improvement in anti-Aspergillus activity when compared with FR131535. However, even though compound 3 showed the lowest MIC value, its in vivo efficacy $\left(\mathrm{ED}_{50} 0.447 \mathrm{mg} \mathrm{kg}^{-1}\right)$ was only slightly better than that of compound $2\left(\mathrm{ED}_{50} 0.658 \mathrm{mg} \mathrm{kg}^{-1}\right)$, which had a 10 -fold greater MIC. This apparent large discrepancy was reduced using serum MIC values that were measured in a medium supplemented with mouse serum. A good correlation between the in vivo effect $\left(\mathrm{ED}_{50}\right)$ and in vitro activity (serum MIC) was obtained. ${ }^{27}$ The marked difference in the MIC values was deduced to result from a decreased concentration of the free form due to binding of the compound to serum protein. On the basis of the observed correlation, prediction of in vivo effects by measuring the serum MIC of the compound became more feasible, and structure-activity relationships were established rapidly. Consequently, compound 3-type derivatives with three linearly linked aromatic rings revealed strong anti-Candida and antiAspergillus activities. However, the hemolytic activity of these analogs was still evident. This was overcome by converting the central benzene ring of compound 3 into various heterocycles. Initially, hemolytic 
activity reduction was difficult because FR901379 derivatives have an amphiphilic structure and surfactant activity. The difference in the amount of unsaturated fatty acids between erythroid and eukaryotic cell membranes was noted, which led to the hypothesis that reduction of hemolytic activity might be possible by decreasing the linearity of the acyl side chains. As expected, the introduction of a heterocycle into the acyl side chain resulted in a marked lowering of hemolytic activity without a decrease in the potent antifungal activity of compound 3. Finally, the cyclic peptide nucleus FR179642, obtained by enzymatic cleavage of the natural product FR901379, was reacylated with an optimal side chain containing an isoxazole ring to yield FK463 (later named micafungin) (Figure 2).

\section{PRE-CLINICAL STUDY OF FK463}

Micafungin is a non-competitive inhibitor of $1,3-\beta$-glucan synthase, a key enzyme necessary for the synthesis of $1,3-\beta$-glucan, that is a main component of fungal cell walls. ${ }^{28-30}$ Fungal cells unable to synthesize $1,3-\beta$-glucan lose the rigidity required to resist osmotic pressure, resulting in cell lysis. This mechanism is common to the echinocandin class of antifungal agents. Most fungal cell walls are composed of chitin, glucan and mannoprotein, although the quantity and relative content of each component depend on fungal species. The multi-enzyme complex 1,3 - $\beta$-glucan synthase is composed of the catalytic subunit FKS1p/FLs2p and the regulatory subunit Rholp GTPase. ${ }^{31}$ The potent inhibitory effect of micafungin on the activity of 1,3- $\beta$-glucan synthase was determined in membrane preparations of both C. albicans and A. fumigatus by measuring incorporation of $\left[{ }^{14} \mathrm{C}\right]$ UDP-glucose into a trichloroacetic-acid-insoluble precipitate. The short-term exposure of C. albicans to $0.1-0.5 \mathrm{mg} \mathrm{ml}^{-1}$ micafungin induced abnormal swelling and irregular shape of fungal cells, a reported feature resulting from inhibition of cell wall synthesis. ${ }^{32}$

Micafungin shows potent fungicidal activity against clinically isolated yeast species of Candida, including C. albicans, C. dubliniensis, C. tropicalis, C. glabrata and C. krusei, and against C. parapsilosis, C. lusitaniae and C. guilliermondii with slightly higher $\mathrm{MIC}_{90}$ values. ${ }^{33-35}$ No cross-resistance to fluconazole-resistant clinical isolates of Candida has been observed. ${ }^{36}$ Characteristically, micafungin has potent in vitro inhibitory activity against Aspergillus species at lower concentrations than amphotericin B and itraconazole, but micafungin was not fungicidal against Aspergillus spp. ${ }^{37}$ There are no reports on acquired resistance to micafungin in susceptible yeast species in clinical practice, except for a case of resistance developed in C. parapsilosis. ${ }^{38}$ Repeated passage of $C$. albicans in sub-inhibitory concentrations of micafungin resulted in negligible changes in MIC values, which suggests a low possibility of developing resistance in a clinical setting.

Among the compounds prepared in the optimization study, micafungin had the strongest in vivo effects against Candida spp. and Aspergillus spp. The efficacy of micafungin was determined in neutropenic murine models of disseminated candidiasis and aspergillosis in comparison with amphotericin B and fluconazole. ${ }^{39}$ As shown in Table 7, the $\mathrm{ED}_{50}$ values of micafungin against disseminated infections with C. albicans, C. glabrata, C. tropicalis and C. krusei ranged from $0.14-0.77 \mathrm{mg} \mathrm{kg}^{-1}$. Although micafungin efficacy was $1.4-3.1$ times inferior to that of amphotericin B $\left(0.09-0.26 \mathrm{mg} \mathrm{kg}^{-1}\right)$, it was $9.6 \geqslant 77$ times superior to that of fluconazole. The $\mathrm{ED}_{50}$ of micafungin against disseminated C. parapsilosis infection was $1.0 \mathrm{mg} \mathrm{kg}^{-1}$, which was 11 times superior to that of fluconazole $\left(10.9 \mathrm{mg} \mathrm{kg}^{-1}\right)$ and 18 times inferior to that of amphotericin B $\left(0.06 \mathrm{mg} \mathrm{kg}^{-1}\right)$. Micafungin was also effective against disseminated $A$. fumigatus infection, with $\mathrm{ED}_{50}$ values in the range of $0.25-0.50 \mathrm{mg} \mathrm{kg}^{-1}$. The efficacies of micafungin were 1.7-2.3 times inferior to those of amphotericin
Table 7 In vivo efficacy of micafungin in a neutropenic mouse model of disseminated candidiasis and aspergillosis

\begin{tabular}{lccc}
\hline \multirow{2}{*}{ Organism } & \multicolumn{3}{c}{$E D_{50}\left(\mathrm{mgkg}^{-1}\right)$} \\
\cline { 2 - 4 } & Micafungin & Fluconazole & Amphotericin B \\
\hline C. albicans FP633 & 0.18 & 1.54 & 0.07 \\
C. glabrata 16011 & 0.18 & 4.89 & 0.10 \\
C. tropicalis 16004 & 0.35 & 7.2 & 0.21 \\
C. krusei 15001 & 1.61 & $>20.0$ & 0.71 \\
C. parapsilosis 16005 & 3.21 & 4.57 & 0.08 \\
C. guilliermondii 13003 & 0.77 & 6.27 & 0.32 \\
A. fumigatus TIMM0063 & 0.33 & $>20.0$ & 0.25 \\
A. fumigatus IFM40835 & 0.26 & $>20.0$ & 0.25 \\
A. fumigatus IFM40836 & 0.45 & $>20.0$ & 0.46 \\
\hline
\end{tabular}

B $\left(0.11-0.29 \mathrm{mg} \mathrm{kg}^{-1}\right)$ and more than 80 times superior to those of fluconazole. These results indicated that micafungin would be a potent parenteral therapeutic agent for disseminated candidiasis and aspergillosis.

\section{CLINICAL STUDY OF FK463}

The clinical efficacy of micafungin was evaluated in the treatment and prophylaxis of Candida and Aspergillus infections. A dose-response trial and two randomized double-blinded trials were first conducted to evaluate the safety and efficacy of micafungin in the treatment of esophageal candidiasis in patients with AIDS. ${ }^{40-42}$ A total of 120 patients with endoscopically proven esophageal candidiasis were included in the initial study, with an i.v. micafungin dosing of 12.5, 50, 75 and $100 \mathrm{mg} \mathrm{day}^{-1}$. Clinical improvement was endoscopically noted in all patients, especially those who received 75 or $100 \mathrm{mg} \mathrm{day}^{-1}$. Symptoms improved or resolved within 3-5 days of treatment in the majority of patients, irrespective of the underlying CD4+ lymphocyte count. The efficacies of i.v. micafungin and i.v. fluconazole were compared in a randomized, double-blind, dose-response study. In all, 245 patients with a prior diagnosis of AIDS/human immunodeficiency virus (HIV) infection and esophageal candidiasis confirmed by endoscopy and culture were randomized to receive micafungin $\left(50,100\right.$ or $\left.150 \mathrm{mg} \mathrm{day}^{-1}\right)$ or fluconazole $\left(200 \mathrm{mg} \mathrm{day}^{-1}\right)$. Both agents were administered once daily by $1-\mathrm{h}$ i.v. infusion for 14-21 days and the response was endoscopicallly determined. Cure rates were 68.8, 77.4 and $89.8 \%$ with 50, 100 and $150 \mathrm{mg}$ of micafungin, respectively. The average cure rate with two higher doses of micafungin $(83.5 \%)$ was comparable to that of $200 \mathrm{mg}$ of fluconazole (86.7\%). This study confirmed the efficacy of micafungin in treatment of esophageal candidiasis in HIV-positive patients.

Other treatment and prophylaxis trials against candidemia, invasive candidiasis and aspergillosis were conducted to assess the efficacy, adverse effects, pharmacokinetics, drug-drug interactions and dosing of micafungin. A convenient dosing regime, an excellent safety profile and remarkably few drug interactions were observed, indicating that micafungin is a potent weapon in antifungal therapy.

\section{OTHER SULFATED ECHINOCANDINS}

The acyl side chain of echinocandin has been readily replaced by established semi-synthesis methods; however, modification of the cyclic peptide by chemical synthesis is difficult. As an alternative, Fujisawa's researchers attempted to obtain structural diversity in the cyclic peptide by extensive screening of fungal products. A number of 


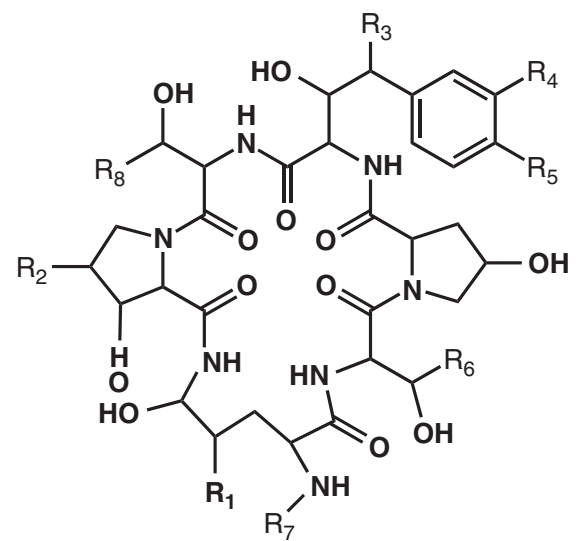

\begin{tabular}{|c|c|c|c|c|c|c|c|c|}
\hline & R1 & R2 & R3 & R4 & R5 & R6 & R7 & R8 \\
\hline FR901379 & $\mathrm{OH}$ & $\mathrm{CH}_{3}$ & $\mathrm{OH}$ & $\mathrm{OSO}_{3} \mathrm{H}$ & $\mathrm{OH}$ & $\mathrm{CH}_{3}$ & Palmitoyl & $\mathrm{CH}_{2} \mathrm{CONH}_{2}$ \\
\hline FR901381 & $\mathrm{OH}$ & $\mathrm{CH}_{3}$ & $\mathbf{H}$ & $\mathrm{OSO}_{3} \mathrm{H}$ & $\mathrm{OH}$ & $\mathrm{CH}_{3}$ & Palmitoyl & $\mathrm{CH}_{2} \mathrm{CONH}_{2}$ \\
\hline FR901382 & H & $\mathrm{CH}_{3}$ & H & $\mathrm{OSO}_{3} \mathrm{H}$ & $\mathrm{OH}$ & $\mathrm{CH}_{3}$ & Palmitoyl & $\mathrm{CH}_{2} \mathrm{CONH}_{2}$ \\
\hline FR220897 & $\mathrm{OH}$ & $\mathrm{H}$ & H & $\mathrm{OH}$ & $\mathrm{OSO}_{3} \mathrm{H}$ & $\mathrm{CH}_{3}$ & Palmitoyl & $\mathrm{CH}_{2} \mathrm{CONH}_{2}$ \\
\hline FR220899 & $\mathrm{OH}$ & $\mathrm{CH}_{3}$ & H & $\mathrm{OH}$ & $\mathrm{OSO}_{3} \mathrm{H}$ & $\mathrm{CH}_{3}$ & Palmitoyl & $\mathrm{CH}_{2} \mathrm{CONH}_{2}$ \\
\hline FR209602 & $\mathrm{OH}$ & $\mathrm{CH}_{3}$ & $\mathbf{H}$ & $\mathrm{OSO}_{3} \mathrm{H}$ & $\mathrm{OH}$ & $\mathbf{H}$ & Palmitoyl & $\mathrm{CH}_{2} \mathrm{CONH}_{2}$ \\
\hline FR209603 & $\mathrm{OH}$ & $\mathbf{H}$ & H & $\mathrm{OSO}_{3} \mathrm{H}$ & $\mathrm{OH}$ & H & Palmitoyl & $\mathrm{CH}_{2} \mathrm{CONH}_{2}$ \\
\hline FR209604 & $\mathbf{H}$ & $\mathrm{CH}_{3}$ & $\mathbf{H}$ & $\mathrm{OSO}_{3} \mathrm{H}$ & $\mathrm{OH}$ & $\mathbf{H}$ & Palmitoyl & $\mathrm{CH}_{2} \mathrm{CONH}_{2}$ \\
\hline FR190293 & $\mathrm{OH}$ & $\mathrm{CH}_{3}$ & $\mathrm{OH}$ & $\mathrm{OSO}_{3} \mathrm{H}$ & $\mathrm{OH}$ & $\mathrm{CH}_{3}$ & Branched acyl & $\mathrm{CH}_{2} \mathrm{CONH}_{2}$ \\
\hline FR227673 & $\mathrm{OH}$ & $\mathrm{CH}_{3}$ & $\mathrm{OH}$ & $\mathrm{OSO}_{3} \mathrm{H}$ & $\mathrm{OH}$ & $\mathrm{CH}_{3}$ & Branched acyl & $\mathrm{CH}_{2} \mathrm{CONH}_{2}$ \\
\hline Echinocandin B & $\mathrm{OH}$ & $\mathrm{CH}_{3}$ & $\mathrm{OH}$ & $\mathbf{H}$ & $\mathrm{OH}$ & $\mathrm{CH}_{3}$ & Linoleoyl & $\mathrm{CH}_{3}$ \\
\hline Aculeacin Ay & $\mathrm{OH}$ & $\mathrm{CH}_{3}$ & $\mathrm{OH}$ & H & $\mathrm{OH}$ & $\mathrm{CH}_{3}$ & Palmitoyl & $\mathrm{CH}_{3}$ \\
\hline Pneumocandin B0 & $\mathrm{OH}$ & $\mathbf{H}$ & $\mathrm{OH}$ & $\mathbf{H}$ & $\mathrm{OH}$ & $\mathrm{CH}_{3}$ & Branched acyl & $\mathrm{CH}_{2} \mathrm{CONH}_{2}$ \\
\hline Mulundocandin & $\mathrm{OH}$ & $\mathrm{CH}_{3}$ & $\mathrm{OH}$ & $\mathbf{H}$ & $\mathrm{OH}$ & $\mathrm{CH}_{3}$ & Branched acyl & H \\
\hline
\end{tabular}

Figure 4 Summary of the structure of echinocandins.

sulfated echinocandins, which differ not only in the amino acid constituents of the cyclic peptide but also in the acyl chain, have been isolated (Figure 4). FR209602, FR209603, FR209604, FR220897 and FR220899 have different methylation and hydroxylation modifications of amino acids in the cyclic peptide, with the same palmitoyl acyl chain as FR901379, ${ }^{43,44}$ whereas FR190293 and FR227673 have the same cyclic peptide as FR901379, with different branched acyl side chains. ${ }^{45}$ Furthermore, the sulfated hydroxyl residue presents at a different position in FR209602, FR209603 and FR209604 than those in other sulfated echinocandins. The strain producing FR209602, FR209603 and FR209604, identified as C. crateriformis no. 738, also belongs to the same genus as that producing FR901379. The strain producing FR220897 and FR220899, identified as C. empetri, belonged to the same species as that producing FR901379. On the contrary, FR190293 and FR227673 were produced from non-Coleophoma strains; that is, Tolypocladium parasiticum and Chalara sp., respectively. Whereas structural diversity in cyclic peptides was observed among the compounds produced by the three strains belonging to the Coleophoma genus, chemical diversity in the acyl side chain was exhibited by non-Coleophoma species.

Considering other echinocandins, we have proposed a classification model for the echinocandin-producing strains, based on the chemical diversity of their product: type I as a sulfated echinocandin-producing strain and type II as a non-sulfated echinocandin-producing strain (Table 8). ${ }^{46}$ Type I has been further classified into two sub-groups. Type IA is a coelomycetes group that produces a sulfated cyclic peptide nuclear structure with a palmitoyl chain. The strains belonging to this group form conidial structures only on leaf segments. Type IB, a hyphomycetes group, produced FR190293 and FR227673, which comprise the same peptide nuclear structure as FR901379, with a variety of branched acyl side chains. These strains form hyphal conidia in a general medium. Type II includes strains (for example, Aspergillus spp. and G. lozoyensis) that produce non-sulfated echinocandins, such as echinocandin B, aculeacin A, mulundocandin and pneumocandin B0. Type I fungi may have a specific enzyme, aryl sulfotransferase, to sulfate a phenolic hydroxyl residue in the cyclic peptide structure. This putative classification model may help in elucidating the molecular evolution of echinocandins, as well as the phylogenetics of the strains producing them.

\section{PROSPECTS}

A novel series of sulfated echinocandins has been reviewed in this paper. The compounds are highly soluble in water, even at a concentration of $50 \mathrm{mg} \mathrm{ml}^{-1}$. Their structural and physico-chemical properties enabled the efficient development of micafungin. Thus, chemical diversity in screening sources is a key factor in successful 
Table 8 A classification model for echinocandin-producing microorganisms

\begin{tabular}{|c|c|c|c|}
\hline Class & $\begin{array}{l}\text { Sub } \\
\text { group }\end{array}$ & Compound & Producing strain \\
\hline $\begin{array}{l}\text { Type I } \\
\text { sulfated }\end{array}$ & & $\begin{array}{l}\text { FR901379 } \\
\text { FR901381 } \\
\text { FR901382 }\end{array}$ & Coleophoma empetri \\
\hline & A & $\begin{array}{l}\text { FR209602 } \\
\text { FR209603 } \\
\text { FR209604 }\end{array}$ & C. crateriformis \\
\hline & & $\begin{array}{l}\text { FR220897 } \\
\text { FR220899 }\end{array}$ & C. empetri \\
\hline & B & $\begin{array}{l}\text { FR190293 } \\
\text { FR227673 }\end{array}$ & $\begin{array}{l}\text { Tolypocladium parasiticum } \\
\text { Chalara sp. }\end{array}$ \\
\hline $\begin{array}{l}\text { Type II } \\
\text { non-sulfated }\end{array}$ & & $\begin{array}{l}\text { Aculeacin Ar } \\
\text { Echinocandin B } \\
\text { Pneumocandin BO } \\
\text { Mulundocandin }\end{array}$ & $\begin{array}{l}\text { Aspergillus japonicus var. aculeatus } \\
\text { A. nidulans var. echinulatus } \\
\text { Glarea lozoyensis } \\
\text { A. sydowi }\end{array}$ \\
\hline
\end{tabular}

drug discovery and subsequent development. In the case of microbial screening, such diversity can be achieved by suitable selection of producing strains and culture conditions. Chemical diversity in the echinocandin family has been observed not only in the cyclic peptide nuclear structure but also in the acyl side chain moiety. Interestingly, various fungi produce common structures, such as an acylated cyclic hexapeptide, with some modifications. Thus far, the mechanism for the biosynthesis of echinocandins has not been elucidated, although it is likely to include a non-ribosomal peptide synthetase to produce the cyclic peptide core, with a fatty acid or polyketide synthase to produce the acyl side chain. An increase in echinocandin chemical diversity may be realized using genetic engineering of the biosynthetic genes.

\section{ACKNOWLEDGEMENTS}

It has been an honor to have been involved in the discovery of micafungin and to be able to present this paper. I express my sincere appreciation to all my colleagues at Fujisawa Pharmaceutical Co., Ltd who participated in the project to discover and develop micafungin. I am also grateful to Professor Satoshi Ōmura for encouraging me to prepare this paper.

1 Clark, T. A. \& Hajjeh, R. A. Recent trends in the epidemiology of invasive mycoses. Curr. Opin. Infect. Dis. 15, 569-574 (2002).

2 Richardson, M. \& Lass-Flörl, C. Changing epidemiology of systemic fungal infections. Clin. Microbiol. Infect. Suppl. 4, 5-24 (2008).

3 Burges, J. L. \& Birchall, R. Nephrotoxicity of amphotericin B, with emphasis on changes in tubular function. Am. J. Med. 53, 77-84 (1972).

4 Carledge, J. D., Midgley, J. \& Gazzard, B. G. Clinically significant azole cross-resistance in Candida isolates from HIV-positive patients with oral candidiasis. AIDS 11, 1839-1844 (1997).

5 Aperis, G. \& Mylonakis, E. Newer triazole antifungal agents: pharmacology, spectrum, clinical efficacy and limitations. Expert Opin. Investig. Drugs 15, 579-602 (2006).

6 Nyfeler, R. \& Keller-Schierlein, W. Echinocandin Bein neuartiges polipeptide-antibiotikum aus Aspergillus nidulans var.echinatus: Isolierung und Bausteine. Helvet. Chim. Acta. 57, 2459-2477 (1974).

7 Schwartz, R. E., Giacobbe, R. A., Boand, J. A. \& Monaghan, R. L. L-671,329, a new antifungal agent. I. Fermentation and isolation. J. Antibiot. 42, 163-167 (1989).
8 Iwamoto, T. et al. WF11899A, B, and C, novel antifungal lipopeptides. I. Taxonomy, fermentation, isolation and physico-chemical properties. J. Antibiot. 47, 1084-1091 (1994).

9 Debono, M. et al. The synthesis and evaluation of LY121019: A member of a series of semi-synthetic analogs of the antifungal lipopeptide echinocandin B. Ann. NY Acad. Sci. 544, 152-167 (1988).

10 Debono, M. et al. Semisynthetic chemical modification of the antifungal lipopeptide echinocandin B (ECB): structure-activity studies of the lipophilic and geometric parameters of polyarylated acyl analogs of ECB. J. Med. Chem. 38, 3271-3281 (1995).

11 Bartizal, K. et al. In vitro preclinical evaluation studies with the echinocandin antifungal MK-0991 (L-743,872). Antimicrob. Agents Chemother. 41, 2326-2332 (1997).

12 Denning, D. W. Echinocandins: a new class of antifungal. J. Antimicrob. Chemother. 49, 889-891 (2002)

$13 \mathrm{Ikeda}, \mathrm{F}$. et al. Role of micafungin in the antifungal armamentarium. Curr. Med. Chem. 14, 1263-1275 (2007).

14 Hope, W. W., Shoham, S. \& Walsh, T. J. The pharmacology and clinical use of caspofungin. Expert Opin. Drug Metab. Toxicol. 3, 263-274 (2007).

15 Chandrasekar, P. H. \& Sobel, J. D. Micafungin: a new echinocandin. Clin. Infect. Dis. 42, 1171-1178 (2006)

16 Fujie, A. Discovery of micafungin (FK453): a novel antifungal drug derived from a naural product lead. Pure Appl. Chem. 79, 603-614 (2007).

17 Yamaguchi, H., Hiratani, T., Iwata, K. \& Yamamoto, Y. Studies on the mechanism of antifungal action of aculeacin A. J. Antibiot. 35, 210-219 (1982).

18 Debono, M. et al. Synthesis of new analogs of echinocandin B by enzymatic deacylation and chemical reacylation of the echinocandin B peptide: synthesis of the antifungal agent cilofungin (LY121019). J. Antibiot. 42, 389-397 (1989).

19 Schmatz, D. M. et al. Pneumocandins from Zalerion arboricola. IV. Biological evaluation of natural and semisynthetic pneumocandins for activity against Pneumocystis carinii and Candida species. J. Antibiot. 45, 1886-1891 (1992).

20 Iwamoto, T., Fujie, A., Nitta, K., Hashimoto, S., Okuhara, M. \& Kohsaka, M. WF11899A, B and C, novel antifungal lipopeptides. II. Biological properties. J. Antibiot. 47, 1092-1097 (1994).

21 Iwamoto, T. Studies on Antifungal Antibiotics Ph.D. Thesis, University of Tokyo (1996).

22 Boeck, L. D., Fukuda, D. S., Abbott, B. J. \& Debono, M. Deacylation of echinocandin B by Actinoplanes utahensis. J. Antibiot. 42, 382-388 (1989).

23 Fujie, A. et al. FR131535, a novel water-soluble echinocandin-like lipopeptide: synthesis and biological properties. Bioorg. Med. Chem. Lett. 11, 399-402 (2001).

24 Tomishima, M., Ohki, H., Yamada, A., Maki, K. \& Ikeda, F. Novel echinocandin antifungals. Part 2: Optimization of the side chain of the natural product FR901379. Bioorg. Med. Chem. Lett. 18, 2886-2890 (2008).

25 Tomishima, M., Ohki, H., Yamada, A., Maki, K. \& Ikeda, F. Novel echinocandin antifungals. Part 1: Novel side-chain analogs of the natural product FR901379. Bioorg. Med. Chem. Lett. 18, 1474-1477 (2008).

26 Tomishima, M. et al. FK463, a novel water-soluble echinocandin lipopeptide: synthesis and antifungal activity. J. Antibiot. 52, 674-676 (1999).

27 Maki, K. et al. Use of a serum-based antifungal susceptibility assay to predict the $i$ $n$ vivo efficacy of novel echinocandin compounds. Microbiol. Immunol. 52, 383-391 (2008).

28 Denning, D. W. Echinocandin antifungal drugs. Lancet 362, 1142-1151 (2003).

29 Kurtz, M. B. \& Douglas, C. M. Lipopeptide inhibitors of fungal glucan synthase. J. Med. Vet. Mycol. 35, 79-86 (1997).

30 Hatano, K., Morishita, Y., Nakai, T. \& Ikeda, F. Antifungal mechanism of FK463 against Candida albicans and Aspergillus fumigatus. J. Antibiot. 55, 219-222 (2002).

31 Qadota, H. et al. Identification of yeast Rholp GTPase as a regulatory subunit of 1,3-beta-glucan synthase. Science 272, 279-281 (1996)

32 Nishiyama, Y., Uchida, K. \& Yamaguchi, H. Morphological changes of Candida albicans induced by micafungin (FK463), a water-soluble echinocandin-like lipopeptide. J. Electron Microsc. 51, 247-255 (2002).

33 Laverdiere, M., Hoban, D., Restieri, C. \& Habel, F. In vitro activity of three new triazoles and one echinocandin against Candida bloodstream isolates from cancer patients. J. Antimicrob. Chemother. 50, 119-123 (2002)

34 Ostrosky-Zeichner, L. et al. Antifungal susceptibility survey of 2,000 bloodstream Candida isolates in the United States. Antimicrob. Agents Chemother. 47, 3149-3154 (2003).

35 Tawara, S. et al. In vitro activities of a new lipopeptide antifungal agent FK463, against a variety of clinically important fungi. Antimicrob. Agents Chemother. 44, 57-62 (2000).

36 Uchida, K., Nishiyama, Y., Yokota, N. \& Yamaguchi, H. In vitro antifungal activity of a novel lipopeptide antifungal agent FK463, against various fungal pathogens. J. Antibiot. 53, 1175-1181 (2000).

37 Nakai, T. et al. In vitro activity of FK463, a novel lipopeptide antifungal agent, against a variety of clinically important molds. Chemotherapy 48, 78-81 (2002).

38 Moudgal, V., Little, T., Boikov, D. \& Vazquez, J. A. Multiechinocandin- and multiazoleresistant Candida parapsilosis isolates serially obtained during therapy for prosthetic valve endocarditis. Antimicrob. Agents Chemother. 49, 767-769 (2005).

39 Chiou, C. C., Mavrogiorgos, N., Tillem, E., Hector, R. \& Walsh, T. J. Synergy, pharmacodynamics, and time-sequenced ultrastructural changes of the interaction between nikkomycin Z and the echinocandin FK463 against Aspergillus fumigatus. Antimicrob. Agents Chemother. 45, 3310-3321 (2001). 
40 Pettengell, K., Mynhardt, J., Kluyts, T., Lau, W., Facklam, D. \& Buell, D. Successful treatment of oesophageal candidiasis by micafungin: a novel systemic antifungal agent. Aliment. Pharmacol. Ther. 20, 475-481 (2004).

41 de Wet, N. et al. A randomized, doubleblind, parallel-group, dose-response study of micafungin compared with fluconazole for the treatment of esophageal candidiasis in HIVpositive patients. Clin. Infect. Dis. 39, 842-849 (2004).

42 de Wet, N. T. et al. A randomized, double blind, comparative trial of micafungin (FK463) vs. fluconazole for the treatment of oesophageal candidiasis. Aliment. Pharmacol. Ther. 21, 899-907 (2005).
43 Kanasaki, R. et al. FR209602 and related compounds, novel antifungal lipopeptides from Coleophoma crateriformis no.738. I. Taxonomy, fermentation, isolation and physico-chemical properties. J. Antibiot. 59, 137-144 (2006).

44 Kanasaki, R. et al. FR220897 and FR220899, novel antifungal lipopeptides from Coleophoma empetri no. 14573. J. Antibiot. 59, 149-157 (2006).

45 Kanasaki, R. et al. FR227673 and FR190293, novel antifungal lipopeptides from Chalara sp. No. 22210 and Tolypocladium parasiticum No. 16616. J. Antibiot. 59, 158-167 (2006).

46 Hino, M. et al. Chemical diversity in lipopeptide antifungal antibiotics. J. Ind. Microbiol. Biotechnol. 27, 157-162 (2001). 